Gdańsk 2019, Nr. 40

https://doi.org/10.26881/sgg.2019.40.06

Agnieszka K. Haas

Universität Gdańsk, Philologische Fakultät /

Uniwersytet Gdański, Wydział Filologiczny

https://orcid.org/0000-0002-2768-5981

\title{
Aufhebung der Weiblichkeit? Pflanzenmetaphorik und Geschlechterdarstellungen in der Literatur um 1800
}

\begin{abstract}
Im vorliegenden Beitrag gilt die Aufmerksamkeit der Pflanzenmetaphorik und ihrem Zusammenhang mit der Geschlechterproblematik in der deutschen Frühromantik. Im Artikel wird hervorgehoben, dass der Pflanzentopos auf die Geschlechterdifferenzen hinweist und mit dem religiös-mystischen Topos der Suche nach dem vollkommenen Menschen verbunden ist. Im Symbol der Pflanze werden beide Geschlechter metaphorisch aufgehoben und versöhnt.
\end{abstract}

Schlüsselwörter: Anthropologie, Geschlecht, Pflanze, blaue Blume, Frühromantik, Georg Philipp Friedrich Freiherr von Hardenberg (Novalis), Karl Wilhelm Friedrich von Schlegel, Johann Wolfgang von Goethe

Transcending of Femininity? Plant Metaphors and Gender Representations in German Literature around 1800. This article discusses the metaphors of plants and their relationship to the gender issue in the early German Romanticism. The article emphasizes that the topos of plant points to differences between man and woman and is linked to the religious-mystical topos of searching for the perfect human being. In the plant symbol, both sexes are metaphorically suspended and reconciled.

Keywords: anthropology, Gender, plant, Blue Flower, Early Romanticism, Georg Philipp Friedrich von Hardenberg (Novalis), Karl Wilhelm Friedrich von Schlegel, Johann Wolfgang von Goethe

Welchem Zweck dient die Suche nach den Affinitäten zwischen Mensch, Pflanze und Tier? Anhand von ihnen versuchte man in der Aufklärung die bisherige Stellung des Menschen im System der Natur mit Hilfe von neuen wissenschaftlichen Entdeckungen zu verifizieren. Die polarisierte Auffassung des Menschen mit seiner Gliederung in Leib und Seele, die auf den Dualismus von Platon zurückgeht, erinnert an eine Pflanze, die sich mit ihren Wurzeln an den Boden hält und ihre Blüte (wie die Seele) gegen den Himmel richtet (Volkmann 2002: 11).

Im naturphilosophischen Gedicht Tier und Pflanze (1802) von Friedrich Wilhelm Joseph Schelling wird der Mensch als geteiltes Wesen gezeigt, dessen „Zeugungskraft“ tierisch, 
das heißt männlich ist und dessen geistige Natur einer Pflanze ähnlich ist, die das Weibliche vertritt:

[...] Pflanze, [...]

Dich verknüpfet der Sonn und dem Reiche des Lichts das Geschlecht nur;

Anders verhält sich das Thier, anders verhält sich der Mensch,

Welcher, sonnengeboren, nur durch das Geschlecht in der Erde

Wurzelnd, den Himmel dadurch zaubert zur Erde herab. (Schelling 1861: 439)

Johann Gottfried Herder, der den Menschen „als Thier und Pflanze“, als „vollkommenste animalische Pflanze" (zit. nach Albus 2001: 301) bezeichnete, hob die privilegierte Stellung des Menschen in der Kette der Wesen hervor, in der die Seele allerdings unberücksichtigt war. Herder stellte zwar die Unsterblichkeit der Seele nicht in Frage, betonte aber oft die Rolle des Körpers, den die Aufklärer nicht mehr cartesianisch behandelten.

Die Pflanzensymbolik des ausgehenden 18. Jahrhunderts entwickelt sich im Schatten der Auseinandersetzung mit der Körperlichkeit und Sinnlichkeit, die ausformuliert werden (Zimmermann 1997: 194). Als unbeherrschbar und „tierisch“ gilt dabei die Sinnlichkeit (des Mannes) (Bergengruen/Borgards/Lehmann 2001: 9), für „pflanzenhaft“ (passiv) wird die Frauennatur gehalten (Balmer 2009: 207).

Während Herder die Pflanze für das Bild des Menschen im Allgemeinen hält und zwischen zwei Geschlechtern nicht unterscheidet, stellt sie Schelling als Symbol der Weiblichkeit und der Geistigkeit dar. Durch das Oxymoron „,animalische Pflanze“ misst Herder dem Menschen aktive und passive Merkmale bei: Der Pflanze kommt das Attribut der Ganzheit zu, ohne dass ihr eine religiöse Färbung verliehen wird. Die Differenzen in Schellings Gedicht ergeben sich aus seiner polarisierten naturphilosophischen Auffassung der vergeistigten Natur, die sich nach Paul Kluckhohn in die „Dreiteilung der Potenzen des Lebens“ verwandelt (Kluckhohn 1961: 26). Dieses polare Ordnungsprinzip betrifft auch beide Geschlechter:

[...] lebendige Einheit in der Zweiheit, [...] sieht Schelling in der ganzen Natur [...], in dem kosmischen Gegensatz von Licht und Schwere sowie auch in dem Gegensatz der Geschlechter, [...] den er auch in dem Gegensatz von Tier als männlich und Pflanze als weiblich wiedererkennen will. (Kluckhohn 1961:25-26)

Analoge Vergleiche ${ }^{1}$ werden in der Poesie und Philosophie angestellt. Nicht selten werden in sie die Entdeckungen der Naturwissenschaften eingebettet. Erkenntnisse der Botanik, Physik, Chemie zusammen mit naturphilosophischen und mystisch-alchemistischen Überzeugungen werden zu metaphorischen Bildern der conditio humana in den literarischen Texten von Goethe, Schlegel oder Novalis. Die traditionelle Pflanzen- und Blumensymbolik, in der die Blüte, Früchte, Stängel, Blätter und Wurzeln für verschiedene Bereiche des menschlichen Handelns stehen, wird in der Romantik mit wissenschaftlichen und naturphilosophischen Inhalten zusammengefügt.

Die vorliegende Skizze hat zum Ziel, die frühromantische Pflanzenmetaphorik in Bezug auf das Menschenbild, den Geschlechterdiskurs und die Kategorie der wechselwirkenden

1 Vgl. dazu. Frevert 1988; Frevert 1995: 13-60. 
Einheit zu analysieren und sie anhand der ausgewählten Romanfragmente von Karl Wilhelm Friedrich von Schlegel (1772-1829) und Georg Philipp Friedrich von Hardenberg (Novalis, 1772-1801) mit den botanischen Erkenntnissen zu konfrontieren sowie mit erotischen und religiösen Bedeutungen der Pflanzenmetapher im Kontext der All-Einheitslehre darzustellen.

\section{Die All-Einheitslehre und ihre Folgen}

In der philosophischen Anthropologie funktionieren verschiedene Modelle des Menschenbildes, die um 1800 relevant sind: 1) das dualistische Konzept der Einteilung in Körper und Geist und der Gliederung der Welt in zwei Bereiche (Pleger 2013: 14), 2) die monistische Überzeugung von der Einheit und dem „Verhältnis des [menschlichen] Teils zum Ganzen" (ebd.) und 3) das Stufenmodell in drei Varianten. 'Stufen', ,Schichten' und ,Kette der Wesen' (ebd.) sind keine Synonyme, denn der geistige Charakter des Kette-Konzepts schließt seine Kompatibilität mit der Evolution aus.

Die romantisch-idealistische Einheitslehre, die auf die Antike und die Frühe Neuzeit zurückgeht, führt zu Versuchen, Differenzen verschiedener Art auszugleichen. Die ihr nah stehende Idee von der ewigen Wiederkehr wird in der Gnosis als eine Triade dargestellt: Einem „anfängliche[n] Zusammensein“ folgt die Trennung, die mit dem „Wiederzusammenfügen“ des Getrennten enden soll (andere Begriffe - „Ganzheit, Chiasma, Rekurs“ - kommen ebenso in Frage, vgl. Aurnhammer 1994: 172). Die Triade wird im Symbol der Pflanze, die sich im Lebenszyklus immer wieder erneuert und eine harmonische Entwicklung durchläuft, auf verschiedene Art und Weise realisiert.

Die Pflanzenmetapher kommt auch im Kontext der Lehre von der einstigen Einheit und ihrer Wiederherstellung vor und beeinflusst in dem Maße den Geschlechterdiskurs um 1800, in dem die gegenseitige soziale Ergänzung der Geschlechter und ihre humanitäre Vervollkommnung an Bedeutung gewinnen. Die heilsgeschichtliche Dimension, die in der Frühromantik populär wird, mythologisiert die Vereinigung von „Weiblichkeit“ und „Männlichkeit" und lässt sie im Kontext der alchemistischen und naturphilosophischen Tradition in ein neues Licht rücken. Das Komplementärverhältnis zwischen den Geschlechtern wird allerdings nicht eindeutig verstanden. In der (metaphorischen oder postulierten) Aufhebung der Geschlechterpolaritäten kommt der Frau eine untergeordnete Stellung zu. Beim Einsatz des Symbols der Pflanze, die im Gegensatz zum Tier als Etikett der (weiblichen) Passivität gilt, ist die starke Differenzierung besonders sichtbar.

\section{Die Botanik um 1800}

Einfluss auf die vegetabile Symbolik um 1800 hatten unter anderem die Botanik und anthropomorphisierte Schilderungen der Physiologie der Pflanzen, die seit der Aufklärung nicht mehr für seelenlose Maschinen gehalten wurden. Ihre Physiologie war allerdings mit irrationalen Überzeugungen aufgeladen. Man schrieb ihnen Empfindungen, Gefühle und Leidenschaften 
zu. ${ }^{2}$ In der Schrift Von den Hochzeiten der Pflanzen (1746) spekulierte Carl von Linné, dass sie Liebeslust empfinden. Früher, in Systema naturae (1735), verglich er das Pflanzenleben mit dem menschlichen Sexualleben und wurde daher der Verunglimpfung „des sittlichen Gefühls"angeklagt (Vietor 2001: 313). Auch in Novalis' Gedicht Astralis, das die Geburt des Astralleibes eines Künstlers darstellt, wird erotisch-botanische Pflanzensemantik eingesetzt.

Versunken lag ich ganz in Honigkelchen.

Ich duftete, die Blume schwankte still

In goldner Morgenluft. (Novalis 1969: 259)

Andere Bezeichnungen wie Narbe, Staubgefäße oder die Vorstellung von der Befruchtungsfeuchtigkeit hat Novalis den botanischen Schriften entnommen (Vietor 2001: 313). Der Bestäubungsprozess der Blüte verläuft hier nach den damaligen botanischen Vorstellungen und dem Konzept Goethes im Lehrgedicht Metamorphose der Pflanzen (Vietor 2001:314). Die botanischen Prozesse der Geburt werden auf das Leben einer „synthetischen Person“, des „Makroanthropos“, übertragen (ebd.: 15).

Die botanischen Ordnungssysteme, die seit der Frühen Neuzeit aufkommen, werden im 17. Jahrhundert auf wissenschaftliche Grundlagen gestellt, was die Botanik jedoch nicht von mythischen und anthropomorphen Darstellungen befreite. Noch in der Hochromantik wird E. T. A. Hoffmann sie in seinen Prosawerken wie Goldner Topf oder Meister Floh gern in Anspruch nehmen.

Die Theorie von der ungeschlechtlichen Vermehrung der Pflanzen fand ihre Anhänger, obwohl R. C. Camerarius im Jahre 1694 bereits das Gegenteil nachgewiesen hatte (Mägdefrau 1992: 136). ${ }^{3}$ Die Überzeugung trug bereits früher zur Entstehung des pflanzlichen Keuschheitssymbols bei, dem die „Bastardbefruchtung im Pflanzenreich“ widersprach (Mägdefrau 1992: 135). Wichtiger scheint die Entdeckung der Monözie, pflanzlicher Zweigeschlechtigkeit, denn sie beeinflusste die romantische Naturphilosophie und wurde auf das literarische Bild des (androgynen) Menschen übertragen (Aurnhammer 1994: 179). Sowohl Goethe als auch Schlegel und Novalis setzen sich mit diesem Phänomen - jeder auf seine Art - auseinander.

\section{Goethe: Metamorphose der Pflanzen}

Epochengeschichtlich gesehen gehört weder Herders noch Goethes Schaffen zur Romantik. Goethes Konzept der Urpflanze ist erwähnenswert, denn seine Abhandlung Versuch

2 In Politia naturae (1760) schreibt ihnen Linné ein Auffassungsvermögen zu (vgl. Ingensiep 1994: 69). Ähnliche Versuche werden von Johann A. Unzer, Schreiben an Mademoiselle R. vom Gefüble der Pflanzen (1766) und Johann Gottlob Krügers Träume (1785) durchgeführt (ebd.).

3 Ch.K. Sprengel hat in seinem Werk Das entdeckte Geheimnis der Natur im Bau und der Befruchtung der Blumen (1793) die Geschlechtlichkeit der Pflanzen empirisch bewiesen (Mägdefrau 1992: 139-141). Diese Erkenntnis wurde aber mehrmals aus philosophischen Gründen geleugnet. Von solchen irreführenden Erkenntnissen der Botaniker wie F. J. Schelver (1812-23), A. W. Henschel (1820) hat sich sogar Goethe überzeugen lassen. Erst dank der Arbeiten von Carl Friedrich Gärtner, der bis Ende der 40er Jahre des 19. Jahrhunderts publizierte, „hat die Diskussion über die Sexualität der Pflanzen ihren Abschluß gefunden“ (Mägdefrau 1992: 138). 
die Metamorphose der Pflanzen zu erklären (1790) und ihre dichterische Version (Metamorphose der Pflanzen, 1799) fanden großen Widerklang bei den Frühromantikern.

Das Urpflanze-Modell diente Goethe zur Erklärung des Urphänomen-Konzepts und der Bildungsgesetze der Natur, die er auf einen parallelen Umwandlungsprozess ,auf einer geistigen Leiter" übertrug, ${ }^{4}$ womit er die religiös-mystische Naturauffassung mit dem Evolutionsmodell (Vietor 2001: 314) vereinen wollte.

In der Elegie Die Metamorphose der Pflanzen (1798) übertrug er den vegetabilen Entfaltungsmechanismus auf das menschliche Leben; der pflanzliche Lebenszyklus wurde repräsentativ für die geistigen Naturgesetze: „Jede Pflanze verkündet dir nun die ewgen Gesetze“ (Goethe 1873: 463). Den Naturgesetzen sollte wiederum die Vervollkommnung im gesellschaftlichen Handeln entsprechen. Alle Evolutionsstufen der Natur waren in der Metamorphose der Pflanzen (und Tiere) sichtbar und auf andere Lebensbereiche, insbesondere auf die Bildung übertragbar. Die wichtigste Rolle in dieser Hierarchie kommt der Liebe zu, die sich ebenfalls stufenweise entwickelt. Die Bekanntschaft und Freundschaft führen zur Liebe, und diese gilt als mythisches Vervollkommnungszeichen.

\section{Zur Entstehungsgeschichte der Romanfragmente von Schlegel (Lucinde) und Novalis (Heinrich von Ofterdingen)}

Im Romanfragment Lucinde (1799), das den Untertitel Bekenntnisse eines Ungeschickten trägt, steht die Liebesbeziehung von Julius und Lucinde im Mittelpunkt. Aus der Vielfalt der Formen wie Briefe, Dialoge, Aphorismen, Tagebucheinträge, die im Roman auftauchen, ergibt sich eine arabeske Struktur des Textes, der über sich reflektiert. Hudgins (1976: 312) zufolge entspricht diese Struktur dem Konzept romantischer Ironie und dem Konstrukt der Urpflanze, das Schlegel von Goethe übernahm. In den im Roman verstreuten Erörterungen zur Geschlechtsproblematik bedient sich Schlegel der Pflanzenmetaphorik, die trotz der Erklärungen im Text nicht eindeutig ist. Seine Metaphern der Sinnlichkeit durchbrachen ein Tabu und sorgten damit für die Kritik an der Unsittlichkeit.

In Lucinde fungiert die Pflanze als Metapher der Frau, der Weiblichkeit im Allgemeinen, der Männlichkeit (!), des Liebespaares, der Menschheit und der Welt. Ansonsten ist das semantische Feld „Pflanze“ in vielerlei Hinsicht mit verschiedenen Ausdrücken besetzt, in denen andere Pflanzenteile (Blätter, Blüten, Früchte, Stängel usw.) oder die ihre Physiologie darstellenden Verben vorkommen.

Schlegels Lucinde und Goethes Metamorphosenlehre wurden von Hardenberg rezipiert, der in seinem Romanfragment Heinrich von Ofterdingen (1802) den Entwicklungsweg des Dichters und damit die Vervollkommnung des Menschengeschlechts thematisierte. Die Pflanzenmetaphorik (allem voran die blaue Blume) wird in Bezug auf die Weiblichkeit, Liebe und Dichtung in Anspruch genommen und in einen heilsgeschichtlichen Kontext gestellt, der die Sphäre der

4 Franz Biet überprüft Goethes Metamorphosenlehre in Bezug auf die Erkenntnisse der Naturwissenschaften des 21. Jahrhunderts (vgl. Biet 2018: 99-135). Biet weist auf die Ähnlichkeiten zwischen dem Modell der Urpflanze und der Struktur der Nukleinsäure hin. 
Sinnlichkeit und Erotik nicht ausschließt. Für das Konzept des Romans und das Geschlechterverständnis waren Schriften von Böhme und Fichte von Bedeutung, die Novalis während des Studiums an der Freiberger Bergakademie las (Furness 1965: 60). In den theoretischen Abhandlungen - im Enzyklopädie-Projekt, im Allgemeinen Brouillon (1798/99) - den Romanfragmenten Die Lebrlinge zu Sais (1798/99) und Heinrich von Ofterdingen, äußert er sich zum männlichen und weiblichen Geschlechtscharakter, den er heilsgeschichtlich wahrnimmt.

Schlegel und Novalis bauen ihre theoretischen Ansätze in die Struktur ihrer Romane ein. Schlegel, der sich mit der Geschlechterproblematik in seinen Abhandlungen Über die weiblichen Charaktere in den griechischen Dichtern und Über die Philosophie. An Dorothea zwischen 1794 und 1799 befasst, bezieht sie auch in das Romanfragment Lucinde ein. ${ }^{5}$

Bereits in der Schrift Über die Diotima (1795) schlug Schlegel eine Komplementarität der Geschlechter und ihr Wechselverhältnis (im engen Sinne der Wortes) vor, indem er eine „selbständige Weiblichkeit“ und „sanfte Männlichkeit“ für möglich hielt (zit. nach Landfester 1997: 100).

Für Novalis' Weiblichkeitsauffassung kann auch eine biographische Begebenheit von Belang sein, nämlich die Begegnung mit der zwölfährigen Christiane Wilhelmine Sophie von Kühn am 17. November 1794, mit der er sich im März des darauffolgenden Jahres verlobte, die kurz danach erkrankte und zwei Jahre später verstarb. Diese Erlebnisse haben womöglich zur Idealisierung der Weiblichkeit beigetragen, die Novalis seither mit Liebe und Tod konnotierte. Zur Herausbildung der Metapher der blauen Blume, über deren Interpretationsmöglichkeiten Herbert Uerlings ausführlich berichtet, ${ }^{6}$ haben mehrere Faktoren beigetragen. Aus Platzgründen wird ihre Analyse auf einige Aspekte eingeschränkt.

\section{Lucinde und Mathilde als Weiblichkeitsideale}

Zur Gleichsetzung des Heiligen mit der Weiblichkeit kommt es im Kapitel Allegorie von der Frechheit, wo der Ich-Erzähler auf die Systematik der Pflanzen anspielt und ihr Lucindes Einmaligkeit gegenüberstellt. Was aus der Frau eine Eingeweihte macht, ist - wie bei Goethe - die Liebe. Die Kritik am aufklärerischen Stufenmodell und an Linnés Systematik sowie an Goethes Bildungsbegriff liegen auf der Hand:

Darum gibt es in der weiblichen Liebe keine Grade und Stufen der Bildung, überhaupt nichts allgemeines [...]. Kein Linné kann uns alle diese schönen Gewächse und Pflanzen im großen Garten des Lebens klassifizieren und verderben; und nur der eingeweihte Liebling der Götter versteht ihre wunderbare Botanik. (Schlegel 1799: 66)

In Lucinde sieht der Erzähler ein Frauenideal, das die blühende Pflanze verbildlicht. Im Kapitel Allegorie von der Frechheit wird wiederum zwischen der Blume als Zeichen der Weiblichkeit und der Pflanze als Symbol der ganzen Menschheit unterschieden:

5 Mit der „Utopie” der Aufhebung der Geschlechterdifferenzen u. a. in Schlegels Lucinde setzt sich Ulrike Landfester (1997: 101) auseinander, die feststellt, dass der „Geschlechtertausch auf der Ebene der Liebeshandlung [...] nur im Rahmen exklusiver Intimität auf der Bühne des Liebesspiels“ zugelassen wird (ebd.: 101).

6 Vgl. dazu Schuth 1995: 133, Schulz 2011: 184, Uerlings 1991: 407-418. 
Laß mich's bekennen, ich liebe nicht dich allein, ich liebe die Weiblichkeit selbst. Ich liebe sie nicht bloß, ich bete sie an, weil ich die Menschheit anbete, und weil die Blume der Gipfel der Pflanze und ihrer natürlichen Schönheit und Bildung ist. (Schlegel 1799: 70)

Ähnlich betrachtete Goethe die Natureinheit, die von einer paradoxen Dialektik beherrscht wird: „Alle Gestalten sind ähnlich und keine gleichet der andern“ (Goethe 1873: 461).

Nach Inge Stephan ist Lucinde „die Summe all der Eigenschaften, die [...] Julius [...] kennen gelernt hat" (Stephan 2001: 206). Ist Lucindes Entsprechung, Mathilde aus Novalis` Roman, auch eine Mannesprojektion?

Vegetabile Metaphern wie die Blume werden in der Kultur des Abendlandes grundsätzlich für eine Chiffre des Weiblichen gehalten. Die Polysemie der Blumensymbolik weist sowohl auf den geliebten Menschen, das weibliche Geschlecht, als auch auf die Vergänglichkeit des Lebens hin. Für die Symbolbildung sind die Schönheit, ihr Aufblühen, die Zartheit und Kurzlebigkeit und ihre relative Seltenheit relevant (Grosse Wiesmann 2012: 56-58). All diese Aspekte scheint Mathilde zu repräsentieren.

Aus der Struktur der beiden Romane ergibt sich, dass die Blume (eigentlich die Blüte) in erster Linie auf die Frauen verweist. Mathilde, aber auch andere Frauen- und Männerfiguren im Roman werden mit der Blumenmetaphorik verknüpft.

Heinrich erblickt die Blume im Traum, nachdem er von einem Fremden davon erzählt bekommen hat:

Endlich wollte er sich ihr nähern, als sie auf einmal, sich zu bewegen und zu verändern anfing; die Blätter wurden glänzender und schmiegten sich an den wachsenden Stängel, die Blume neigte sich nach ihm zu, und die Blütenblätter zeigten einen blauen ausgebreiteten Kragen, in welchem ein zartes Gesicht schwebte. (Novalis 1969: 132)

Als er Mathilde kennen lernt und beschreiben will, konzentriert er sich auf ihr Gesicht, ihre Augen und die blauen Adern, für die er farbige Parallelen unter den Gestirnen (Sterne, Sonne) findet. Mathilde selbst vergleicht sich mit einer welkenden Rose, die ihren Tod zu erahnen scheint: „Ach! Heinrich, du weißt das Schicksal der Rosen; wirst du auch die welken Lippen, die bleichen Wangen mit Zärtlichkeit an deine Lippen drücken? Werden die Spuren des Alters nicht die Spuren der vorübergegangenen Liebe sein?" (Novalis 1969: 230).

Der anthropomorphe Charakter der Blume und ihre blaue Farbe könnten an das kränkliche Mädchen Sophie mit seinem blassen, bläulichen Teint erinnern. Sie stehen auch für die unerreichbare, religiös-mystisch gefärbte Sehnsucht. Dietmar Schuth hält die blaue Farbe für „höchst androgyn[]“ (Schuth 1995: 18). Bereits Giese (1919) schreibt der Blume die Androgynität zu, in der sich die Aufhebung der Gegensätze vollzieht, die das Ziel verfolgt, die künftige Vervollkommnung des Menschen (im goldenen Zeitalter) zu erlangen.

Vereint die Blume das männliche und weibliche Prinzip? Erfüllt Novalis dabei den Wunsch, der in Lucinde ausgedrückt wird? „Oder soll es etwa statt dieser bunten Fülle nur eine vollkommne Blume geben, welche alle Schönheiten der übrigen vereint und ihr Dasein überflüssig macht?" (Schlegel 1799: 271).

Aus der Haltung der blauen Blume in der ersten Traumvision geht hervor, dass sie neben der Blüte „Blätter“ und „Stängel“ hat, die sich bewegen. Für das Weibliche steht ihre Blüte, 
die sich in ein Gesicht verwandelt. Das Vorhandensein von anderen Organen legt nahe, dass der männliche Aspekt in der Blume vertreten ist. Diese Züge kommen während ihrer Bewegung ans Licht. Dass sich die romantische Pflanzenmetaphorik ansonsten auf die Männlichkeit im Allgemeinen beziehen kann, bezeugt das folgende Fragment aus Schlegels Lucinde: „Die männliche Ungeschicktheit ist ein mannigfaltiges Wesen und reich an Blüthen und Früchten jeder Art“" (Schlegel 1799: 270-271).

In Novalis' Blume wird die Weiblichkeit (wie die Männlichkeit) zugunsten einer metaphysischen Synthese von Realität und Traum aufgehoben. Die Vereinigung von Dichtung, Liebe und Natur soll ein Zeichen dieses Zustands sein, der im Astralis-Gedicht zum Ausdruck kommt:

Eins in allem und alles im Einen

Gottes Bild auf Kräutern und Steinen

Gottes Geist in Menschen und Tieren,

Dies muß man sich zu Gemüte führen.

Keine Ordnung mehr nach Raum und Zeit

Hier Zukunft in der Vergangenheit. (Novalis 1969: 260)

Die Jenaer Romantiker haben den Androgynie-Mythos unter anderem in der Pflanzenmetapher wiederbelebt, in der die Geschlechterproblematik im gleichen Ausmaß zum Tragen kommt. Aurnhammer (1994: 178) zufolge findet das Androgynie-Motiv Bestätigung in den Wissenschaften (unter anderem in der Botanik) und wird von Novalis zugleich von „heilsgeschichtliche[n] Projektionen“ begleitet (ebd.: 179).?

\section{Liebesakt als Pflanzenbild}

Aurnhammer (1994: 180) zufolge verbildlicht Julius „seine mannweibliche Einheit mit Lucinde $[. .$.$] in der Monözie“. Im Gegensatz zu Schlegels Roman, in dem die Einheit als$ Ziel der Beziehung (und der vereinigten Menschheit) angesehen wird, schildert Goethe in Wilhelm Meisters Lehrjahre die zweigeschlechtliche Pflanze als Allegorie des Anfangs von einem inzestuösen Verhältnis:

Seht die Lilien an: entspringt nicht Gatte und Gattin auf Einem Stengel? Verbindet beide nicht die Blume, die beide gebar, und ist die Lilie nicht das Bild der Unschuld, und ihre geschwisterliche Vereinigung nicht fruchtbar? (zit. nach Aurnhammer 1994: 179)

Geistige Verwandtschaft zwischen den Liebenden ist die Voraussetzung der körperlichen Vereinigung, die im Bild der Pflanze auftaucht. Das Paar wird als pflanzlicher Organismus angesehen:

7 Das Androgynie-Motiv kommt bereits in Goethes Roman Wilhelm Meisters Lebrjabre vor, der im gleichen Maße Schlegels Roman Lucinde geprägt hat. Die Androgynie-Motivik ist jedoch bei Goethe „nicht mit den traditionellen mythologischen Argumenten, sondern mit einer botanischen Erklärung" (Aurnhammer 1994: 179) fundiert. 
Weißt du noch, wie der erste Keim dieses Gedankens vor dir in meiner Seele aufsproßte und auch gleich in der deinigen Wurzel faßte? - So schlingt die Religion der Liebe unsre Liebe immer inniger und stärker zusammen [...]. (Schlegel 1799: 25-26)

Schlegel greift den Topos der miteinander verwachsenen Pflanzen auf, die eine ewige, über das Grab hinausgehende Liebe bedeuten. Das einander liebende Paar steht für die Vollendung der Menschlichkeit, in der die Sinnlichkeit und Geistigkeit im Einklang stehen: „Ich sehe hier eine wunderbare sinnreich bedeutende Allegorie auf die Vollendung des Männlichen und Weiblichen zur vollen ganzen Menschheit" (Schlegel 1799: 28). Die Pflanzenmetaphorik betrifft nicht nur das Pflanzenbild als vereinigtes Paar. Die Physiologie und Metamorphose der Pflanzen werden auf den Gedankengang der Protagonisten übertragen („der erste Keim“, „aufsprossen“, „Wurzel fassen“).

Die Dithyrambische Fantasie über die schönste Situation in Schlegels Lucinde stellt die Wechselwirkung zwischen Mann und Frau in einem Liebesakt auf ähnliche Weise dar. In den Mittelpunkt rückt die geistige Bindung und das Frauenbild, die insgesamt in einer Anamnesis angeschaut werden, in der platonischen Erinnerung an die Vergangenheit, aber auch an die Zukunft. Bei dieser Anschauung müssen alle Zeit- und Raumeinschränkungen verschwinden:

Wir beide werden noch einst in Einem Geiste anschauen, daß wir Blüthen Einer Pflanze oder Blätter Einer Blume sind, und mit Lächeln werden wir dann wissen, daß was wir jetzt nur Hoffnung nennen, eigentlich Erinnerung war. (Schlegel 1799: 25)

Die Elemente der Pflanze oder der Blume (Blüten, Blätter) stellen eine organische Ganzheit dar, die im Bewusstseinszustand rezipiert wird und die „Hoffnung“ und „Erinnerung“ als gleichbedeutende Faktoren der Wirklichkeit, nämlich Zukunft und Vergangenheit, sehen lässt. Die Zeitgrenze wird aufgehoben. Das Gesehene kann sich sowohl auf einen zeitlosen Bewusstseinsprozess als auch auf einen idealen (künftigen?) Zustand beziehen. Einen ähnlichen Gedanken der Alleinheit finden wir im zitierten Fragment des Astralis-Gedichts.

Das von Lucinde repräsentierte Weiblichkeitsideal ist mit Harmonie- und Einheitsvorstellungen verbunden, in denen die Physiologie der Pflanzen ebenso vorkommt:

Aber die volle Harmonie fand er [...] allein in Lucindens Seele, wo die Keime alles Herrlichen und alles Heiligen nur auf den Strahl seines Geistes warteten, um sich zur schönsten Religion zu entfalten. (Schlegel 1799: 210)

Führt der Bezug auf die Religion und die unio mystica zur Idealisierung der beiden Geschlechter, wenn die sakrale Bedeutung mit der Körperlichkeit verknüpft wird? Diese Synthese wird durch das Zusammenfügen von Licht- und Pflanzenmetaphorik erreicht, in der die Wechselwirkung zwischen Lucinde und Julius zustande kommt. Botanische Begriffe (,Keime‘, ,entfalten') werden auf den Anfang ,alles Heiligen“ übertragen, der einen mystifizierten (und mystischen) Liebesakt darstellt. Die Passivität des weiblichen Prinzips wird allerdings beibehalten, das wiederum vom (männlichen) Licht „bestrahlt“ werden muss. Es liegt auf der Hand, dass im Gegensatz zu Schelling das geistige (Licht-)Prinzip eindeutig dem Mann zugeschrieben wird. Andererseits trägt Lucinde einen Namen, dessen Etymologie auf das Licht 
(lat. lux, lucis) verweist. Damit wird die Differenz zwischen ihnen metaphorisch aufgehoben. Nicht zufälligerweise kommt das im Akt des Sprechens bzw. Schreibens zustande, der die Unterschiede auf die Diskursebene übertragen lässt.

Durch die religiösen Anspielungen im Kontext des Liebesaktes scheint die Sinnlichkeit einen transzendenten Charakter zu bekommen. Gleich aber verliert sie ihn. Im Gegensatz zum Begriff der Liebe bei Platon, nach dessen Auffassung diese einen „Aufstieg von der Körperwelt zu den Ideen“ darstellte, spiegelt sich bei Schlegel die „Transzendenz in die Immanenz" (Dehrmann 2017: 177) zurück. Im Liebesakt wird die Sehnsucht aufgehoben, und weil sie „in der Welt ihre Erfüllung“ (Dehrmann 2017: 177) findet, werden dadurch die beiden Geschlechter sowie die Körperlichkeit im Allgemeinen vergeistigt.

Auch bei Goethe, der die Pflanzen und ihren Entwicklungsprozess anthropomorphisiert und mythologisiert, unterliegt die Liebe als mythisch-mystische Kraft einem geistigen Prozess. Die Blüte wird zur Krönung der pflanzlichen Entfaltung und somit zum Symbol der Vollkommenheit, deren höchste Stufe die Liebesvereinigung ist:

die heilige Liebe

Strebt zu der höchsten Frucht gleicher Gesinnungen auf,

Gleicher Ansicht der Dinge, damit in harmonischem Anschaun

Sich verbinde das Paar, finde die höhere Welt. (Goethe 1873: 463)

Die Liebe als Höhepunkt der Humanität erinnert an das gnostische Konzept der himmlischen Hochzeit (bieros gamos), die im alchemistischen Prozess eine Vervollkommnung der Menschheit darstellt, in der die Grenzen aufgehoben werden:

Traulich stehen sie nun, die holden Paare, beisammen,

Zahlreich reihen sie sich um den geweihten Altar. (Goethe 1873: 462)

\section{Erotische Anziehungskraft der blauen Blume}

Dass die Bewegungen der Pflanze in Heinrich Ofterdingen einen erotischen Charakter haben, kommt in der Traumvision, in der der Titelprotagonist in einer unterirdischen Felsenhöhle badet, zum Tragen. Astrid Herbold (2004: 106-107) hebt die Erotik der „Selbstumarmung“" hervor, die sie für ein Sinnbild der Selbstreflexivität des gesamten Romans hält (ebd.: 106) und die sie mit der Umarmung der Mutter nach dem Erwachen verknüpft, die ein „Teil der Blume und der Geliebten" sei (ebd.). ${ }^{8}$

Die erotische Anziehungskraft der (androgynen) Blume lässt sich im Erlebnis des Fremden bemerken, es handelt sich nämlich um eine „,seltsame[] Leidenschaft“: , [...] in der Welt, in der ich sonst lebte, wer hätte da sich um Blumen bekümmert, und gar von einer so seltsamen Leidenschaft für eine Blume hab“ ich damals nie gehört" (Novalis 1969: 130).

8 Die Selbstumarmung der Blume kann mit der Selbstbefruchtung im Gedicht Astralis und dem von Novalis transzendental betrachteten Akt der Selbstliebe zusammenhängen (Vietor 2001: 316). Harald Neumeyer (2001: 95) ist der Meinung, dass die blaue Blume autoreferentiell, als „Sehnsucht nach Onanie“ zu verstehen ist. 
Im Klingsohr-Märchen wird die Erotik in verschiedenen Formen stärker akzentuiert und allegorisiert. Der Zusammenhang zwischen Erotik und Poesie, der in Schlegels Lucinde gleichwohl vorkommt, stellt einen semantischen Höhepunkt im Roman von Novalis dar. Das Motiv der Umarmung, das sowohl als Geste des Androgynen wie auch als Aufhebung der Grenze zwischen einzelnen Wesen verstanden werden kann, kommt im Märchen ebenfalls vor:

Ein Lilienblatt bog sich über den Kelch der schwimmenden Blume; die kleine Fabel saß auf demselben, und sang zur Harfe die süßesten Lieder. In dem Kelche lag Eros selbst, über ein schönes schlummerndes Mädchen hergebeugt, die ihn fest umschlungen hielt. Eine kleinere Blüte schloß sich um beide her, so daß sie von den Hüften an in eine Blume verwandelt zu sein schienen. (Novalis 1969: 242)

Die Liebesvereinigung kann mit der religiös gefärbten Dialektik gleichgesetzt werden, denn „im Akt der Vereinigung [...] sind Vereinzelung und Fremdsein aufgehoben. Darin wächst der Mensch über sich hinaus und empfindet, sei es auch nur als momentan Mögliches, Befreiung und Unendlichkeit" (Schulz 2011: 184). Es ist nicht wegzuleugnen, dass die blaue Blume sowohl die geistig-seelische als auch die erotische Sphäre repräsentiert, die sich nicht ausschließen, sondern eine Einheit anstreben.

Bereits die Erotisierung der ersten Szene führt zur Dimension des Unbewussten, in dem sich Unterschiede aufheben. Die Verbindung zwischen dem Traum und der Dichtung als höchster Handlung führt zur Ästhetisierung der Erlebnisse. Der Wachzustand und Traumzustand, zwischen denen Heinrich balanciert, sind nicht als Gegensätze aufzufassen, sondern als Bestandteile des Ganzen.

Diese Interpretation kann durch das folgende Fragment aus Hardenbergs Fragmente und Studien 1797-1798 bestätigt werden: „,...] Streben nach Einheit - Streben nach Mannigfaltigkeit - durch wechselseitige Bestimmung beider durch einander - wird jene höhere Synthesis der Einheit und Mannigfaltigkeit selbst hervorgebracht - durch die Eins in Allem und Alles in Einem ist" (Novalis 1969: 401-401).

Die dreiteilige Struktur der Märchen-Geschichte, die die Zustände der Harmonie, des Todes und der Flut bis zum vollkommenen mythischen goldenen Zeitalter schildert, entspricht der gnostischen Triade, die oben erwähnt wurde. Die Synthese betrifft das männliche und weibliche Prinzip, das in einem erwarteten und wiederkehrenden mythischen Zeitalter vereint werden soll. Schlegel - wie erwähnt - projiziert diese geistig-körperliche Einheit auf den Akt der Liebe, der in der Gegenwart zustande kommt.

\section{Bewusstseinsprojektionen als Verbildlichung der All-Einheit}

Lässt sich der androgyne Charakter der blauen Blume mit den Projektionen des Bewusstseins vereinen? Die Ähnlichkeit zwischen Mathildes ,schwebendem“ Kopf und der Bewegung der Blume lässt sie in einer dynamischen Wechselwirkung erscheinen, die im Bewusstsein des sie betrachtenden Heinrich zustande kommt:

Auf einem lichthimmelblauen Grunde lag der milde Glanz der braunen Sterne. Stirn und Nase senkten sich zierlich um sie her. Eine nach der aufgehenden Sonne geneigte Lilie war ihr Gesicht, und von dem schlanken, weißen Halse schlängelten sich blaue Adern in reizenden Windungen um die zarten 
Wangen. Ihre Stimme war wie ein fernes Echo, und das braune lockige Köpfchen schien über der leichten Gestalt nur zu schweben. (Novalis 1969: 211)

Diese Wechselwirkung wird durch den Gebrauch von synonymen oder bedeutungsverwandten Verben erreicht. Den Verben aus der Traumvision („sich bewegen“, „verändern“, „sich schmiegen“, „sich zuneigen“, „schweben“), entsprechen Verbformen wie „sich senken“, „neigen“, „sich schlängeln“, „schweben“. Die Analogie wird deutlicher, als Heinrich nach der Bedeutung seines Traumes zu fragen beginnt: „Welcher sonderbare Zusammenhang ist zwischen Mathilden und dieser Blume? Jenes Gesicht, das aus dem Kelche sich mir entgegenneigte, es war Mathildens himmlisches Gesicht" (Novalis 1969: 218).

Die wichtigste Projektion des Weiblichkeitsideals und der Dichtung ist Mathilde. Als Tochter des Dichters Klingsohr scheint sie zwei Aspekte von Heinrichs Entwicklung - Dichtung und Liebe - in sich zu vereinen. Aber andere Frauenfiguren sind mit der Symbolik der blauen Blume und mit Mathilde ebenso verbunden: die Mutter, Zulima, Veronica, Ginnistan und die Fabel bilden eine Synthese des Weiblichen und der Dichtung, des Realen und des Mythischen.

Diese Harmonie wird polarisiert, indem zum Beispiel Mathilde der lebenslustigen Veronica gegenübergestellt wird, deren lockere Verhaltensweise im Widerspruch zu ihrer Zurückhaltung steht. Es ist kein Zufall, dass Veronica ein Pflanzenname ist und an das Vergissmeinnicht (die blaue Blume) erinnert. Mathildes Tod bedeutet ihre Transzendierung zum Ideal des Weiblichen und der Dichtung. Eine Parallele zu ihrem Tod bildet der Tod der Mutter im Klingsohr-Märchen, deren Asche und kannibalische Verzehrung durch alle wiederum eine Synthese durch den Tod bedeutet. In der Asche sind außerdem keine Differenzierungen mehr sichtbar.

Es scheint, dass sowohl Hardenberg als auch Schlegel die Weiblichkeit synthetisch auf das subjektive Bewusstsein beziehen. In Lucinde wird nämlich „Weiblichkeit auf einmal [...] gesehen“, sie wird „mit dem Auge“ des Geistes gesehen: „die Eine ewig und einzig Geliebte in vielen Gestalten, bald als kindliches Mädchen, bald als Frau in der vollen Blüte [...] und der Weiblichkeit, und dann als würdige Mutter [...]“ (Schlegel 1799: 6).

Durch die Vielheit der Gestalten wird bei Novalis das Prinzip der Poetisierung der Welt realisiert. Sind in dieser Synthese auch männliche Figuren des Romans inbegriffen? Silke Horstkotte zufolge repräsentieren die Frauen das, woran es Heinrich mangelt (2004: 224). Damit werden sie „auf die Funktion eines Anderen des männlichen Subjekts beschränkt“ (ebd.). Die männlichen Protagonisten stehen im gleichen Maße dafür, woran es dem zukünftigen Dichter mangelt. Die Vereinigung mit dem Weiblichen ist ein poetischer Akt; „ein Androgyn-Werden“ beschränkt sich auf den „männlichen Dichter“ und seinen eventuellen Narzissmus (ebd.). Es sei betont, dass ein Androgyn-Werden bereits in die Symbolik der blauen Blume eingeschrieben wird, die als Pflanze „männliche“ Elemente besitzt.

Die Hybride von menschlichen und pflanzenhaften Zügen stellt die Idee des Ganzen, der Aufhebung der Grenzen und der ersehnten All-Einheit dar. Sie wird zugleich auf die Geschlechterdifferenzen projiziert, die in der ideellen Welt verschwinden sollen. Die Blume hat eine doppelte Identität, in der Gegensätze sich ergänzen und aufgehoben werden. Das gilt auch für die Geschlechter, die sie repräsentiert. Der Zustand, in dem die gewünschte Vollkommenheit 
der Ganzheit und Einheit möglich ist, wird von Novalis vor allem auf das Bewusstsein übertragen. Nicht zufälligerweise sieht Heinrich das im Kragen der Blume schwebende Gesicht im Traum, der ein todesähnlicher Bewusstseinszustand ist, in dem Gegensätze verschwinden und das Reale mit dem Unrealen verknüpft wird.

\section{Geistige und ethische Vervollkommnung der Menschheit}

Die Pflanze betrachtet Schlegel als Höhepunkt der geistigen Entwicklung:

[...] je göttlicher ein Mensch oder ein Werk des Menschen ist, je ähnlicher werden sie der Pflanze; diese ist unter allen Formen der Natur die sittlichste, und die schönste. Und also wäre ja das höchste vollendetste Leben nichts als ein reines Vegetieren. (Schlegel 1799: 87; hervorgeh. von dem Autor)

Vietor zufolge könnte diese Stelle Novalis veranlasst haben, „die Idee der Poesie im Bild der ,blauen Blume darzustellen“ (Vietor 2001: 314). Im Allgemeinen Brouillon hat Hardenberg die Blüte als „Symbol des Geheimnisses unseres Geistes“(Vietor 2001:315) beschrieben und sich "die geistige Generation nach dem Modell der pflanzlichen Metamorphose“ vorgestellt.

Bei Schlegel und Novalis ist das pflanzlich-weibliche Idealmodell religiös besetzt (Brauers 1996: 159) und in eine universale Erlösungsphantasie integriert: „Die Welt im Ganzen und ursprünglich ist eine Pflanze und soll auch wieder ganz Pflanze werden“" (zit. nach Brauers 1996:159). Sie ist der Höhepunkt der geistigen Entwicklung: „Ich sehe hier eine wunderbare sinnreich bedeutende Allegorie auf die Vollendung des Männlichen und Weiblichen zur vollen ganzen Menschheit“ (Schlegel 1799: 28).

Der vegetabile Charakter der einzelnen Frau und der Frauen im Allgemeinen wird vor allem in der Liebe und der biologischen Reifung offenbar, die mit den pflanzlichen Geschlechtsorganen verglichen werden:

Auch das Mädchen weiß [...] schon alles, noch ehe der Blitz der Liebe in ihrem zarten Schoß gezündet, und die verschloßne Knospe zum vollen Blumen kelch der Lust entfaltet hat. Und wenn eine Knospe Gefühl hätte, würde nicht das Vorgefühl der Blume deutlicher in ihr sein, als das Bewußtsein ihrer selbst? (Schlegel 1799: 66)

Als Basis für Schlegels Geschlechterverständnis diente ihm, wie erwähnt, die Philosophie von Platon, die auf einem Bezugssystem basierte, in dem die „Ergänzung der Geschlechter“ und der Androgynie-Mythos eine bedeutende Rolle spielen (vgl. dazu Sotzek 2014: 77). Die Geschlechterfrage ist bei ihm in botanische und naturphilosophische Erkenntnis eingebunden. Aus diesem Grunde ist der Rollenwechsel der Geschlechter, den Schlegel in Lucinde vorschlug, im Kontext der platonischen Lehre zu verstehen. Laut Ulrike Landfester sei er utopisch, denn er sei „auf der Ebene der Liebeshandlung [...] nur im Rahmen exklusiver Intimität auf der Bühne des Liebesspiels“ möglich (Landfester 1997: 102). Die Aufhebung der Geschlechterdifferenzen wäre also nur „im Akt sexueller Vereinigung“(ebd.) möglich. Landfester schreibt in diesem Kontext von einer Variante des Rollentausches und einem „Paradox gegenseitiger Aneignung und Abgrenzung“ (ebd.: 103). Es sei hinzugefügt, dass diese Aufhebung in der Gegenwart paradoxerweise nur als vorübergehender Zustand gelten 
sollte, denn das Erreichen vollkommener Harmonie konnte für Romantiker nur eine Annäherung (an das Absolute) sein.

Es soll auch nicht vergessen werden, dass für die religiös-mythischen Vorstellungen der Geschlechterdifferenz bei Schlegel und Novalis neben Platons Gastmabl auch die Böhme-Rezeption und die mit ihr zusammenhängende gnostisch-alchemistische Lehre der Frühen Neuzeit von Bedeutung waren (Furness 1965: 58).

Die Gnosis von Böhme, die Alleinheitslehre und das Androgynie-Motiv werden von Novalis miteinander verknüpft:

In Novalis, who studied Boehme and alchemy extensively, the craving for fusion of world and imagination, self and Sophie, is strongly reminiscent of the desire for androgyny; the yearning for self-identity of subject and object rejects the dichotomy inherent in Fichte's metaphysics and finds the transfiguration of the world in a movement of the heart prompted by all-embracing love. (Furness 1965: 60)

Ruben Zimmermann bemerkt, dass die Untergliederung des Androgynie-Mythos nach Claude Lévi-Strauss die folgenden Kernbegriffe aufweist: „die Einheit, Trennung oder Sehnsucht“ (Zimmermann 2000: 272), wobei „die Beziehung der Menschen zu den Göttern“ eng in den Mythos verwoben ist (ebd.). In Frage kommt hier auch die gnostische Triade EinheitTrennung-Wiederzusammenfügen (Aurnhammer 1994: 172), die auch bei Novalis wegen ihrer heilsgeschichtlichen Bedeutung relevant ist.

Furness zufolge ist die Androgynität ein perfekter Zustand der Harmonie, in dem alle Gegensätze aufgehoben werden (Furness 1965: 58). Die Auffassung der Geschlechter hat bei Schlegel und Novalis einen religiösen, vielleicht auch gnostischen Charakter, der eine genderorientierte Interpretation erschweren kann (vgl. Landfester 1997), denn der Einfluss der Gnosis und der Alchimie trägt zur Metaphorisierung aller Dimensionen des Lebens bei: „for the alchemists sulphur and mercury that is, the male and female principles, was looked upon as being an important step towards the final purification of the material world" (Furness 1965: 60).

Aus den angeführten Beispielen ergibt sich, dass der Androgynie-Mythos, der in die Pflanzenmetaphern eingebettet ist, nicht auf dem ersten Zustand der Triade („Einheit“) basiert, sondern auf die letzte Etappe („Rekurs“) ausgerichtet ist, die sowohl die Zukunft, das goldene Zeitalter, als auch einen Bewusstseinszustand bedeuten kann. Infolge dessen wird die Pflanzenmetapher mit der religiös-ethischen Bedeutung aufgeladen, die dem Mythos ursprünglich fehlte, und in deren Mittelpunkt die Vervollkommnung der Menschheit - durch die (geistige und körperliche) Liebe - rückt.

\section{Literatur}

Albus, Vanessa (2001): Weltbild und Metapher. Untersuchungen zur Philosophie im 18. Jahrhundert. Würzburg: Königshausen \& Neumann.

Aurnhammer, Achim (1994): Die eins waren, eins sind oder eins sein mochten. In: Hartmut Meesmann (Hg.): Androgyn: jeder Mensch in sich ein Paar!? Weinheim: Dt. Studien-Verlag, 171-184.

Balmer, Susanne (2009): Der weibliche Entwicklungsroman als widerspenstiges Narrativ - Pflanzenmetaphorik und bürgerliche Geschlechterdichotomie in „Jülchen Grünthal“ und „Christa 
Ruland“. In: Christa Binswanger u. a. (Hgg.): Gender Scripts. Widerspenstige Aneignungen von Geschlechternormen. Frankfurt: Campus, 205-225.

Bergengruen, Maximilian / Borgards, Roland / Lehmann, Johannes Friedrich (2001): Die Grenzen des Menschen. Anthropologie und Ästhetik um 1800. Einleitung. In: Dies. (Hgg.): Die Grenzen des Menschen. Anthropologie und Ästhetik um 1800. Würzburg: Königshausen \& Neumann, 7-14.

Biet, Franz (2018): Goethe - Aufklärer oder Esoteriker? Wie modern ist Goethe? Studien zu seiner fortdauernden Aktualität. Innsbruck: Studienverlag.

Brauers, Claudia (1996): Perspektiven des Unendlichen: Friedrich Schlegels ästhetische Vermittlungstheorie. Berlin: Erich Schmidt.

Breidbach, Olaf (2001): Transformation statt Reihung - Naturdetail und Naturganzes in Goethes Metamorphosenlehre. In: Ders., Paul Ziche (Hgg.): Naturwissenschaften um 1800. Wissenschaftskultur in Jena-Weimar. Weimar: Böhlau.

Bühler, Benjamin (2013): Botanik. In: Roland Borgards u. a. (Hgg.): Literatur und Wissen. Ein interdisziplinäres Handbuch. Stuttgart: Metzler, 64-69.

Butzer, Günter / Jacob, Joachim (2012): Metzler Lexikon literarischer Symbole, 2. Auf., Stuttgart: Metzler.

Dehrmann, Mark-Georg (2017): Lucinde. In: Johannes Endres (Hg.), Friedrich Schlegel-Handbuch: Leben - Werk - Wirkung. Stuttgart: Metzler, 171-178.

Frevert, Ute (1988): Bürgerliche Meisterdenker und das Geschlechterverhältnis. Konzepte, Erfahrungen, Visionen an der Wende vom 18. zum 19. Jahrhundert. In: Dies. (Hg.), Bürgerinnen und Bürger. Geschlechterverhältnisse im 19. Jahrhundert. ZwölfBeiträge. Göttingen: Vandenhoeck \& Ruprecht, $17-48$.

Frevert, Ute (1995): „Mann und Weib, und Weib und Mann“. Geschlechter-Differenzen in der Moderne. München: C. H. Beck.

Furness, Raymond (1965): The Androgynous Ideal. Its Significance in German Literature. In: The Modern Language Review, 60 (1), 58-64.

Giese, Fritz (1919): Der romantische Charakter. Bd. 1: Die Entwicklung des Androgynenproblems in der Frühromantik. Langensalza: Wendt und Klauwell.

Goethe, Johann Wolfgang von (1873): Gedichte von Goethe. 3. Aufl. Berlin: Grote.

Grosse Wiesmann, Hanna (2012), Blume [Artikel]. In: Günter Butzer, Joachim Jacob (Hgg.): Metzler Lexikon literarischer Symbole. 2. Aufl. Stuttgart: Metzler, 56-58.

Herbold, Astrid (2004): Eingesaugt \& Rausgepresst: Verschriftlichungen des Körpers und Verkörperungen der Schrift. Würzburg: Königshausen \& Neumann.

Horstkotte, Silke (2004): Die Poetik der Androgynie in Novalis“ „Heinrich von Ofterdingen“. In: Herbert Uerlings (Hg.), Novalis. Poesie und Poetik. Tübingen: Niemeyer, 221-240.

Hudgins, Esther (1976): Das Geheimnis der Lucinde-Struktur. Goethes Die Metamorphose der Pflanzen. In: The German Quarterly, 49 (3), 295-311.

Ingensiep, Hans, Werner (1994): Der Mensch im Spiegel der Tier- und Pflanzenseele. Zur Anthropomorphologie der Naturwahrnehmung im 18. Jahrhundert. In: Hans-Jürgen Schings (Hg.): Der ganze Mensch. Anthropologie und Literatur im 18. Jahrhundert. Stuttgart: Metzler, 54-79. Kluckhohn, Paul (1961): Das Ideengut der deutschen Romantik. 4. unveränderte Auflage. Tübingen: Niemeyer. 
Landfester, Ulrike (1997): „Sie und kein anderer war Romeo“. Anmaßung und Freiheit der Frau in Männerkleidung in der Literatur der Kunstperiode. In: Walter Hinderer u. a. (Hgg.): Codierungen von Liebe in der Kunstperiode. Würzburg: Königshausen \& Neumann, 85-112.

Mägdefrau, Karl (1992): Geschichte der Botanik. Leben und Leistung großer Forscher. 2. Aufl., Unveränderter Nachdruck 2013. Berlin: Springer.

Neumeyer, Harald (2001): „Ich bin einer von denjenigen Unglückseligen [...]“. Rückkopplungen und Autoreferenzen. Zur Onaniedebatte im 18. Jahrhundert. In: Maximilian Bergengruen u. a. (Hgg.): Die Grenzen des Menschen. Anthropologie und Ästhetik um 1800. Würzburg: Königshausen \& Neumann, 65-95.

Novalis (1969) Werke. Herausgegeben und kommentiert von Gerhard Schulz. München: C. H. Beck. Pleger, Wolfgang (2013): Handbuch der Anthropologie. Die wichtigsten Konzepte von Homer bis Sartre. Darmstadt: Wissenschaftliche Buchgesellschaft.

Schelling, Friedrich Wilhelm Joseph von (1861): Friedrich Wilhelm Joseph von Schellings sämmtliche Werke. 1. Abt., Bd. 10. Stuttgart: Cotta.

Schlegel, Friedrich von (1799): Lucinde. Ein Roman. Erster Theil. Berlin: Frölich.

Schulz, Gerhard (2011): Novalis: Leben und Werk Friedrich von Hardenbergs. München: C. H. Beck.

Schuth, Dietmar (1995): Die Farbe Blau: Versuch einer Charakteristik. Münster: LIT.

Sotzek, Nicole (2014): Geschlecht als Methode: Androgynie-Konzepte im frühromantischen Geschlechterdiskurs bei Friedrich Schlegel und Friedrich von Hardenberg. Dissertation. Zürich.

URL: https://www.zora.uzh.ch/id/eprint/152967/1/152967.pdf [Zugriffsdatum: 19.07.2019].

Stephan, Inge (2001): Kunstepoche. In: Wolfgang Beutin u. a. (Hgg.): Deutsche Literaturgeschichte. Von den Anfängen bis zur Gegenwart. 6. Aufl. Stuttgart: Metzler, 182-230.

Uerlings, Herbert (1991): Friedrich von Hardenberg, genannt Novalis. Werk und Forschung. Stuttgart: Metzler.

Vietor, Sophia (2001): Astralis von Novalis: Handschrift - Text - Werk. Würzburg: Königshausen \&Neumann.

Volkmann, Helga (2002): Märchenpflanzen, Mythenfrüchte, Zauberkräuter. Grüne Wegbegleiter in Literatur und Kultur. Göttingen: Vandenhoeck \& Ruprecht.

Zimmermann, Harro (1997): Das Projekt Mündigkeit. Kleines Plädoyer für mehr Aufklärung unter Aufklärern. In: Das achtzehnte Jahrhundert. Mitteilungen der Deutschen Gesellschaft für die Erforschung des achtzehnten Jahrhunderts, 21 (2). Wolfenbüttel: Wallstein, 189-201.

Zimmermann, Ruben (2000): Struktur und Kontextualität des Androgynie-Mythos. Zur Mythenhermeneutik von Claude Lévi-Strauss. In: Richard Grathoff, Bernhard Waldenfels (Hgg.): Bildersprache verstehen. Zur Hermeneutik der Metapher und anderer bildlicher Sprachformen. Mit einem Geleitwort von H.-G. Gadamer [Reihe: Übergänge. Texte und Studien zu Handlung, Sprache und Lebenswelt, Bd. 38]. München: Fink, 259-292. 